\title{
A chaotic microresonator structure for an optical implementation of an artificial neural network
}

Phang, Sendy, Bienstman, Peter, Seddon, Angela, Mellor, Chris, Benson, Trevor

Sendy Phang, Peter Bienstman, Angela Seddon, Chris Mellor, Trevor M. Benson, "A chaotic microresonator structure for an optical implementation of an artificial neural network," Proc. SPIE 11283, Integrated Optics: Devices, Materials, and Technologies XXIV, 1128313 (25 February 2020); doi: $10.1117 / 12.2548716$

SPIE. Event: SPIE OPTO, 2020, San Francisco, California, United States 


\title{
A chaotic microresonator structure for an optical implementation of an artificial neural network
}

\author{
Sendy Phang ${ }^{\mathrm{a}}$, Peter Bientsman ${ }^{\mathrm{b}}$, Angela Seddon ${ }^{\mathrm{a}}$, Chris Mellor ${ }^{\mathrm{c}}$, and Trevor M. Benson*a \\ ${ }^{a}$ George Green Institute for Electromagnetics Research, Faculty of Engineering, University of \\ Nottingham, University Park, Nottingham NG7 2RD, UK \\ ${ }^{\mathrm{b}}$ Ghent University imec, Department of Information Technology, Technologiepark-Zwijnaarde \\ 126, 9052 Gent, Belgium \\ cSchool of Physics and Astronomy, University of Nottingham, University Park, Nottingham \\ NG7 2RD, UK
}

\begin{abstract}
Tuneable all-optical signal processing has been the holy grail of information photonics; it has been pursued for many years but has proven to be very challenging. In this contribution, we present our recent work in developing an all-optical signal processing device called a photonic reservoir computer (PhRC) which can be tuned to perform a bespoke task. The PhRC is inspired by how the brain handles and process information. We demonstrate that a chaotic micro-resonator is a suitable platform for the optical implementation of such an artificial neural network.
\end{abstract}

Keywords: All-optical signal processing, photonic reservoir computer, integrated photonics, micro-resonator, mid-infrared spectroscopy.

\section{INTRODUCTION}

Rapid progress in Mid-Infrared (MIR) optics in the last decade has led to commercial MIR optical components, including low-loss chalcogenide-fiber (1.5-10 $\mu \mathrm{m})$, fiber combiners/splitters (1.5-6.5 $\mu \mathrm{m})$ (IRflex Corp), mercurycadmium-telluride (MCT) photodetectors $(2-11 \mu \mathrm{m})$ (ThorLabs), source pigtailing, etc. MIR integrated photonic technology such as silicon-on-sapphire, ${ }^{1}$ germanium-on-silicon (Ge-on-Si),${ }^{2}$ sub-wavelength suspended silicon, ${ }^{3}$ and so on have opened a way of fabricating low loss compact MIR optics in planar technologies.

Artificial intelligence and machine learning have provided headline-making success in the diagnosis of cancer based on image processing. ${ }^{4,5}$ Furthermore, MIR imaging ${ }^{6}$ and spectroscopy ${ }^{7,8}$ based on supercontinuum and narrow-band sources epitomize a new era in healthcare and clinical diagnostics. MIR investigation provides additional rich chemical analysis based on the specific spectral fingerprints of molecular species. It relies on the recognition of molecular-signatures in MIR radiation after tissue-interaction for distinguishing healthy and non-healthy tissue. Fourier-transform infrared (FTIR) based spectro-microscopy technology combines the spatial resolution of optical microscopy with the spectral selectivity of vibrational spectroscopy. Multivariate statistical analysis of such FTIR spectra ${ }^{7}$ shows that the differences in the responses of diseased and healthy tissues are often subtle and may involve hundreds of important spectral fingerprints. Thus, rapid and high-volume information processing is required to perform the necessary spectral pattern recognition if the significant potential of MIR spectroscopy is to be realized as a clinical evidence-based diagnosis aid. The ambitious challenge is to use spectroscopic imaging provide surgeons with an on-the-spot molecular-pathology as a chemical tissue-map. Here we demonstrate that integrated photonics optics is promising exploratory ground for the physical implementation of an Artificial Neural Network (ANN) concept called a Reservoir Computer (RC). ${ }^{9-14}$ The Photonic Reservoir Computer (PhRC) discussed in this paper can be trained to undertake bespoke information processing tasks. It thus provides an important and timely means by which to achieve the signal processing tasks required by MIR spectro-microscopy all-optically using existing and emerging integrated MIR photonics technology platforms.

Further author information: (Send correspondence to T.M.B. or S.P.)

T.M.B.: E-mail: trevor.benson@nottingham.ac.uk; phone +44 115 9515589;

S.P.: E-mail: sendy.phang@nottingham.ac.uk; phone +44 115748 4947;

https://www.nottingham.ac.uk/research/groups/ggiemr/index.aspx 


\section{PHOTONIC RESERVOIR COMPUTING}

\subsection{Introduction to Photonic Reservoir Computing}

This paper demonstrates the application of a chaotic microcavity structure to serve as reservoir-neuron kernel in a Reservoir Computer (RC) algorithm that mimics the complex interconnections of a biological neural network, see Fig. 1. In principle, the RC algorithm exploits the chaotic and echo characteristics of complex neural networks to allow separation in the higher-dimensional parameter space, in the present context it helps achieve the desired separation and identification of the subtle difference in the fingerprint spectra of diseased and healthy tissue. The RC differs from a traditional Recurrent Neural Network (RNN) by embracing randomness. In the $\mathrm{RC}$ approach, a distinction is made between the systems kernel and the read-out layer. In the RC approach, the training phase finds the optimum read-out weights only whilst retaining the characteristic of the RCs kernel as a randomly interconnected recurrent neural network. Consequently, typical RC implementations allow a high degree of freedom in the complicated topology of the neuron kernel. Moreover, integrated optics implementations of the read-out weighting process can be envisioned. ${ }^{15}$ A RC not only provides a distinct approach, but its performance is comparable to, or for some applications even better than, other ANN approaches. ${ }^{9-13}$ We refer the reader to $[9,13]$ for a detailed comparison between Reservoir Computing and Recurrent Neural Networks, and the implementation of the $\mathrm{RC}$ optimization algorithm based on Tikhonov regularization with a cross-validation method

Photonic implementations of a Reservoir Computer (RC) have become popular in recent years. The unique training approach in the RC offers two principal benefits: (1) a feasible physical implementation of an ANN and (2) task-specific training that is done exclusively at the Read-Out layer; this is significantly simpler than the whole system optimization of a Recurrent Neural Network (RNN). Notable Photonic RC developments in different topologies include chains of Semiconductor Optical Amplifiers (SOAs), ${ }^{13}$ chains of micro-ring resonators, ${ }^{16}$ non-linear delay-coupled photonic systems, ${ }^{17}$ and quarter-billiard cavities,${ }^{11}$ for spoken-digit and bit pattern recognition.

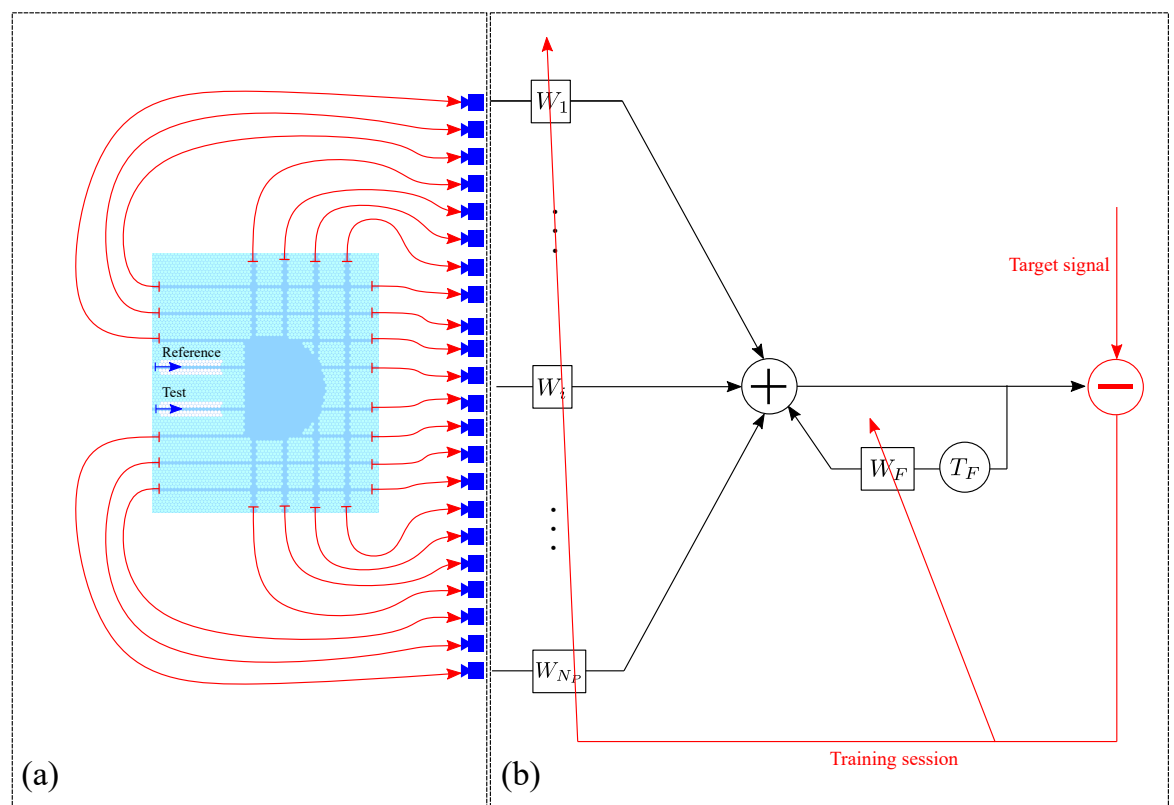

Figure 1. Schematic illustration of photonic reservoir computer. (a) a chaotic photonic crystal microcavity as reservoir kernel, (b) schematic of the recurrent read-out layer of the reservoir computer. In (b), the signal flow direction in solid red coloured line are performed during training session which optimise the read-out weight $W_{1} \cdots N_{p}$ and feedback weight $W_{F} \cdot T_{F}$ denotes the feedback time-delay parameter. 


\subsection{Illustrative example studied}

The example studied employs a D-shaped integrated optics cavity as the neuron kernel to perform temporal signal fingerprinting, targeting MIR bio-chemical spectral fingerprint recognition. It operates at MIR wavelengths and so takes advantage of the rapid developments in MIR photonics technologies. The new diagnostic Photonic Reservoir Computer approach explored is performed directly on temporal signals, instead of the indirect spectral finger-printing as for example in. ${ }^{18}$ Before describing the temporal-signal diagnostic approach used, we shall first describe the sensing element and our implementation of a physical neuron kernel. The results of time-domain simulations based on a commercial implementation of the Finite-Difference Time-Domain (FDTD) method ${ }^{19}$ provide the training dataset. These simulations were performed in two-dimensions with an out-of-plane principal electric field polarisation, i.e. TM-polarised in a conventional two-dimensional problem sense.

Fig. 1(a) illustrates the sensor and the D-shaped optical cavity which serves as the photonic neuron kernel. In order to illustrate of the disease-healthy tissue discrimination concept, a reference 'normal' sample is embedded within some of the air holes on one channel and a test (either normal or contaminated) sample on the other channel. In this illustrative example photonic crystal $(\mathrm{PhC})$ waveguides act as our sensor element; in principle one can use any type of sensor available. The D-shaped cavity is a chopped-circle structure with a radius of 20 $\mu \mathrm{m}$ with input ports connected to the sensor and 22 output ports. In detail, both structures are implemented within a triangular lattice of an air-hole $\mathrm{PhC}$ on a silicon material base whose refractive index is taken to be $n=3.5$. For 2.5 to $2.7 \mu \mathrm{m}$ operation, we use a lattice constant of $r=0.48 a$, where the lattice constant $a=1.3$ $\mu \mathrm{m}$ and $r$ is the radius of the air hole, noting that the $\mathrm{PhC}$ has a full TM band-gap region over the wavelength range from 2.42 to $2.7 \mu \mathrm{m}$.

FDTD simulations were used to model the reservoir kernel and calculate the signal transmission of the structure in Fig. 1(a). The incident signal used was a continuous-wave (CW) at $2.6 \mu \mathrm{m}$ superimposed on which were CW-modulated-Gaussian pulses centred at different wavelengths and peaking at different time, see Fig. 2(a). The spectrum of the incident signal, in Fig. 2(b), depicts eight narrowband non-overlapping Gaussian profiles and a Dirac delta function at the $2.6 \mu \mathrm{m}$ wavelength of the constant $\mathrm{CW}$ signal. The incident signal was launched simultaneously from both sensor channels and its temporal signal transmission was recorded during the FDTD simulation. Considering future implementation of such a system in practice, the photodetectors of the reservoir activation signal (i.e. the temporal transmitted signal of the D-shaped cavity and interconnected waveguide network) will have a sampling capacity limited by their bandwidth and a limitation in the detection of fluctuation in intensity imposed by noise. Therefore, we performed Hilbert transformations on these signals to obtain signal envelopes and allowed for a down-sampling due to sensor sampling bandwidth, here is assumed to be an ambitious 100 Gbps. An example of such reservoir activation states and its envelope is shown in Fig. 2(c).

To obtain the appropriate weight of the read-out layer, these signals (the envelope and the down-sampled reservoir activation signal) and the target signal (i.e. the teacher) were fed into an optimisation algorithm based on Tikhonov regularisation regression with cross-validation method. The reader is referred to ${ }^{10,14}$ for details of the reservoir computer algorithm and the implementation of regression approach. The target signal is determined a priori depending on the sample object placed in the test chamber. If the test chamber is filled with a normal or healthy sample, the target signal is a constant signal of unit amplitude one. In the presence of a contaminated or diseased sample in the test chamber the target signal is set to be a Gaussian pulse biased at one, whose peaks are separated temporarily to correspond with the fingerprint wavelength of the contaminant i.e. at 2.56 and $2.63 \mu \mathrm{m}$ in this illustrative example, see Fig. 2(d). In principle, one has the freedom to choose the target signal; another appropriate target signal function may lead to a better performance and this will be part of our future investigations.

Due to the feedback characteristic of the read-out layer, and the freedom of choosing the target signal, during the training session two tuning parameters namely (i) feedback delay time $T_{F}$ and (ii) target signal time-shift parameter $T_{T}$ were optimized to minimise error. The target signal time-shift parameter $T_{T}$ shifts the temporal position of the target signal with respect to the incident signal, see Fig. 2(d). Here, the Normalised Means Square Error (NMSE) is used as the error figure,

$$
\text { NMSE }=\frac{\left\langle\left(y_{\text {obtained }}-y_{\text {target }}\right)^{2}\right\rangle}{\left\langle y_{\text {target }}^{2}\right\rangle}
$$


(a)

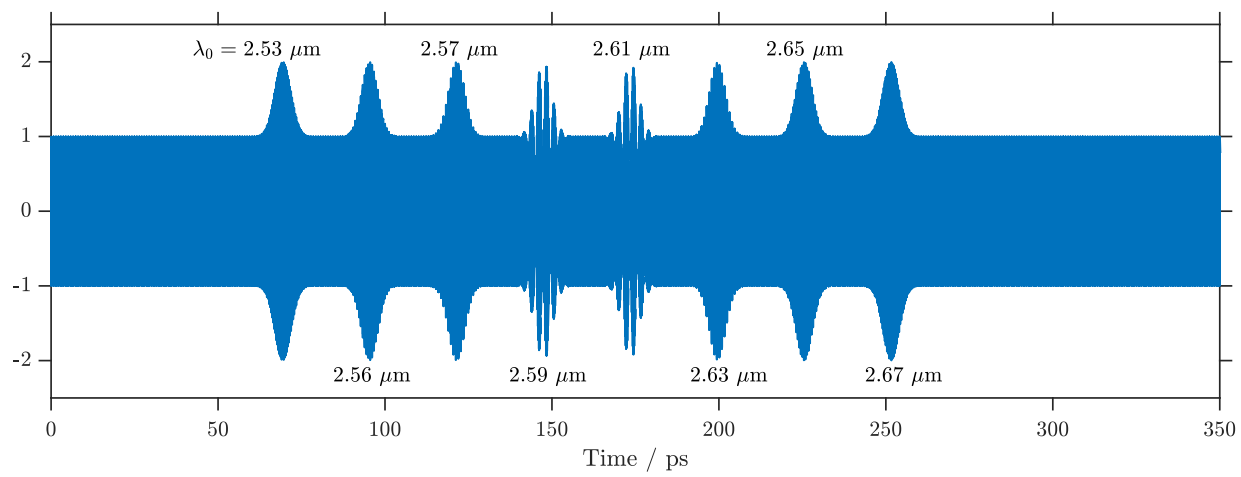

(b)

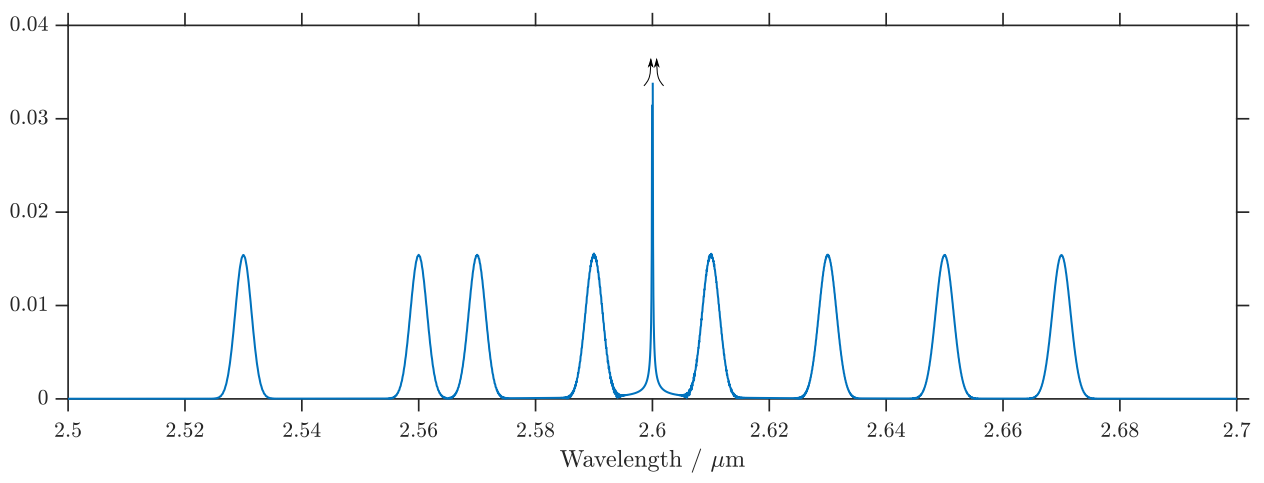

(c)

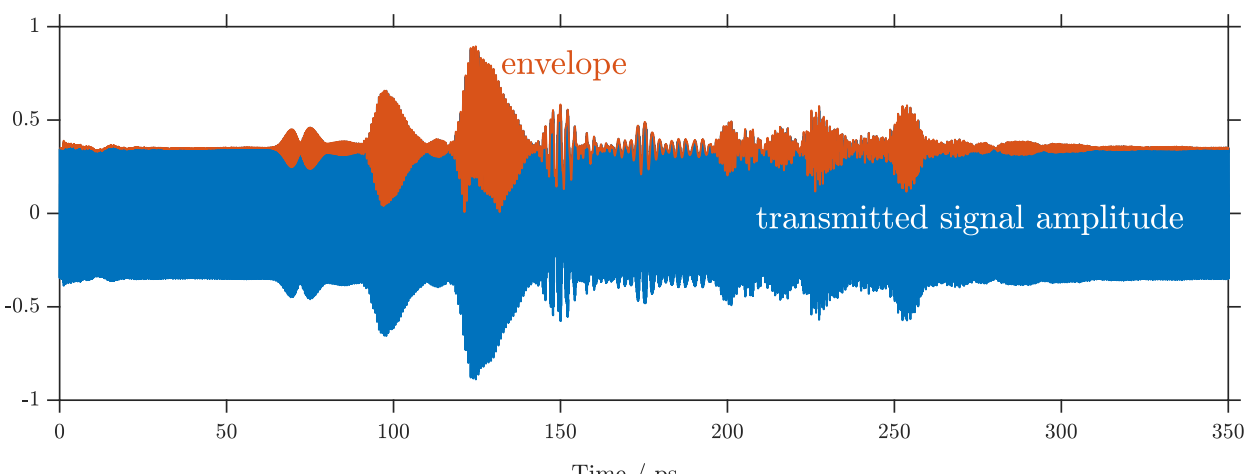

(d)

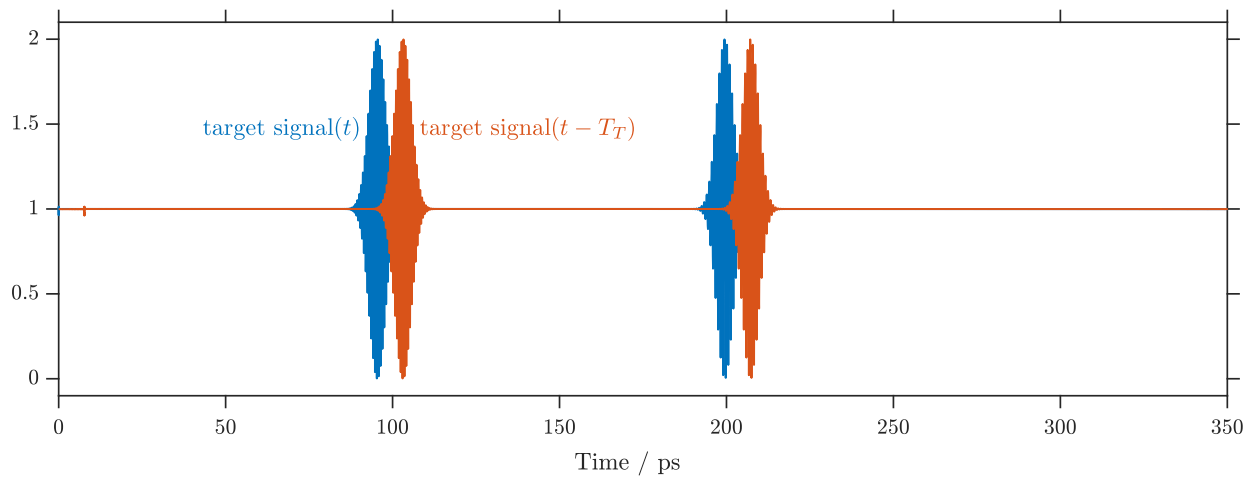

Figure 2. (a,b) Incident signal as function of (a) time and (b) wavelength; (c) typical example of the reservoir kernel activation signal (D-shaped cavity) output, which exhibits strong transient response of the cavity; (d) target signal with and without time-delay parameter $T_{T}$. 

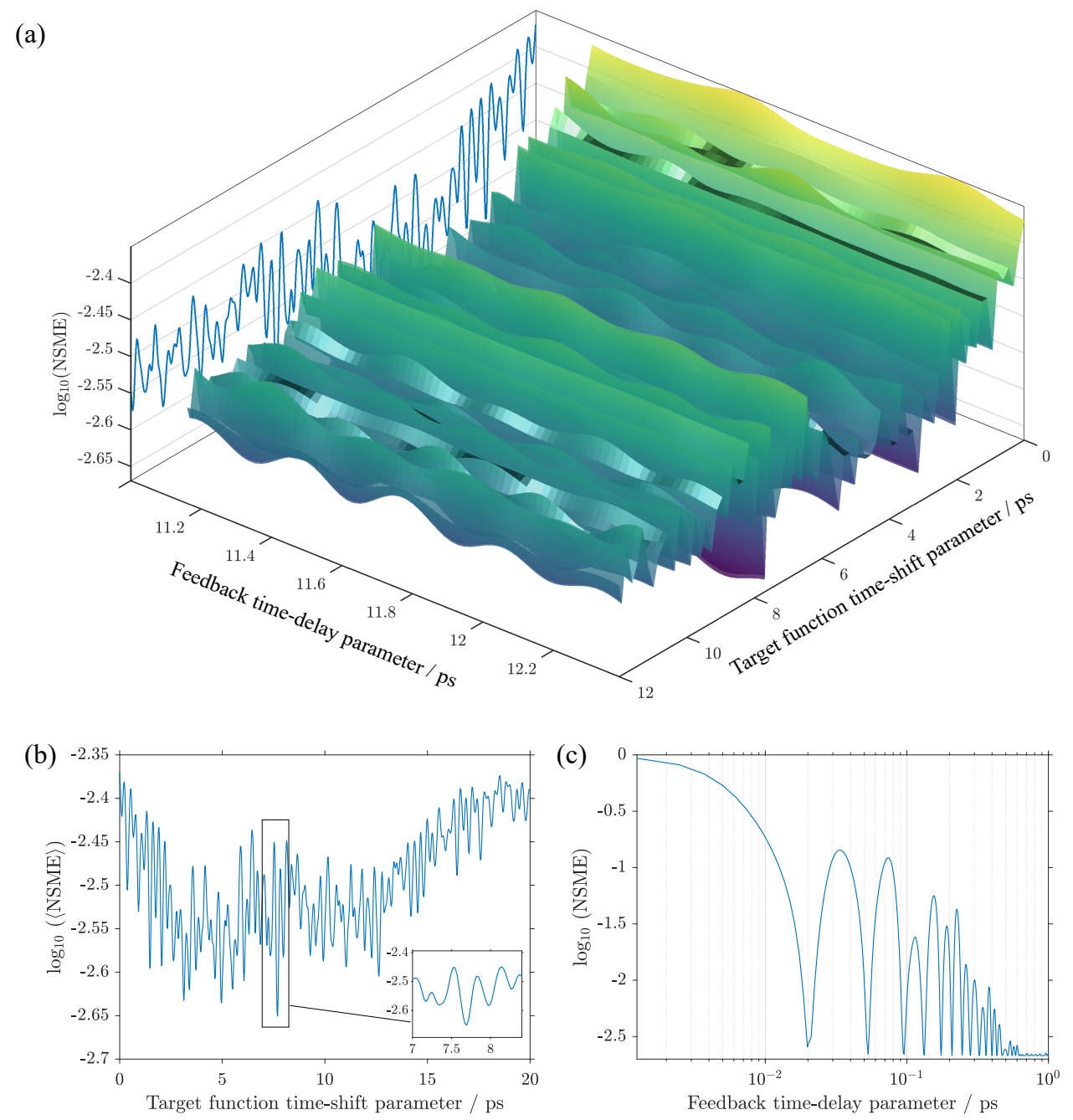

Figure 3. (a) NSME figure of the photonic reservoir computer as a function of the feedback time-delay $T_{F}$ and target function time-shift $T_{T}$ parameters. The ensemble average along the $T_{F}$ direction is plotted as a solid line on the face of the plot (a); for clarity this is re-plotted as subplot (b). (c) NSME plot as function of the $T_{F}$ parameter at $T_{T}=7.7$ ps.

where $\langle\cdot\rangle$ it the ensemble average operation, $y_{\text {target }}$ and $y_{\text {obtained }}$ denote the target signal and the obtained reservoir output signal, respectively.

Figure 3(a) shows the NSME as function of the two tuning parameters. The NSME is observed to fluctuate weakly in the feed-back time-delay parameter $T_{F}$ direction in comparison to the target function time-shift parameter $T_{T}$. To find the optimum tuning parameters, we followed a two-stage parameter sweep procedure. First, we performed a parameter sweep of $T_{T}$ for several different values of $T_{F}$ and averaged the NSME as depicted in Fig. 3(b). The inset to Fig. 3(b) shows the slowly varying NSME; with minimum NSME within the range $0<T_{T}<20 \mathrm{ps}$ is found at $7.7 \mathrm{ps}$. Using the optimum target function time-shift parameter $T_{T}=7.7$ ps, a second parameter sweep was then performed for the feedback time-delay parameter $T_{F}$. The NSME as function of $T_{F}$, in Fig. 3(c), shows that there exist multiple optimum $T_{F}$ values. Although Fig. 3(c) shows that the general trend of NMSE is decreasing and plateauing to a certain value of NSME, we believe that this trend will no longer be the case for large values of $T_{F}$. It seems rationale that at very large $T_{F}$ (far beyond the transient response of the reservoir) the feedback will either have no contribution (has zero weight) or at least an immaterial contribution. We are, currently, investigating the relationship between the tuning parameter $T_{F}$ and the decay time of the reservoir kernel. 

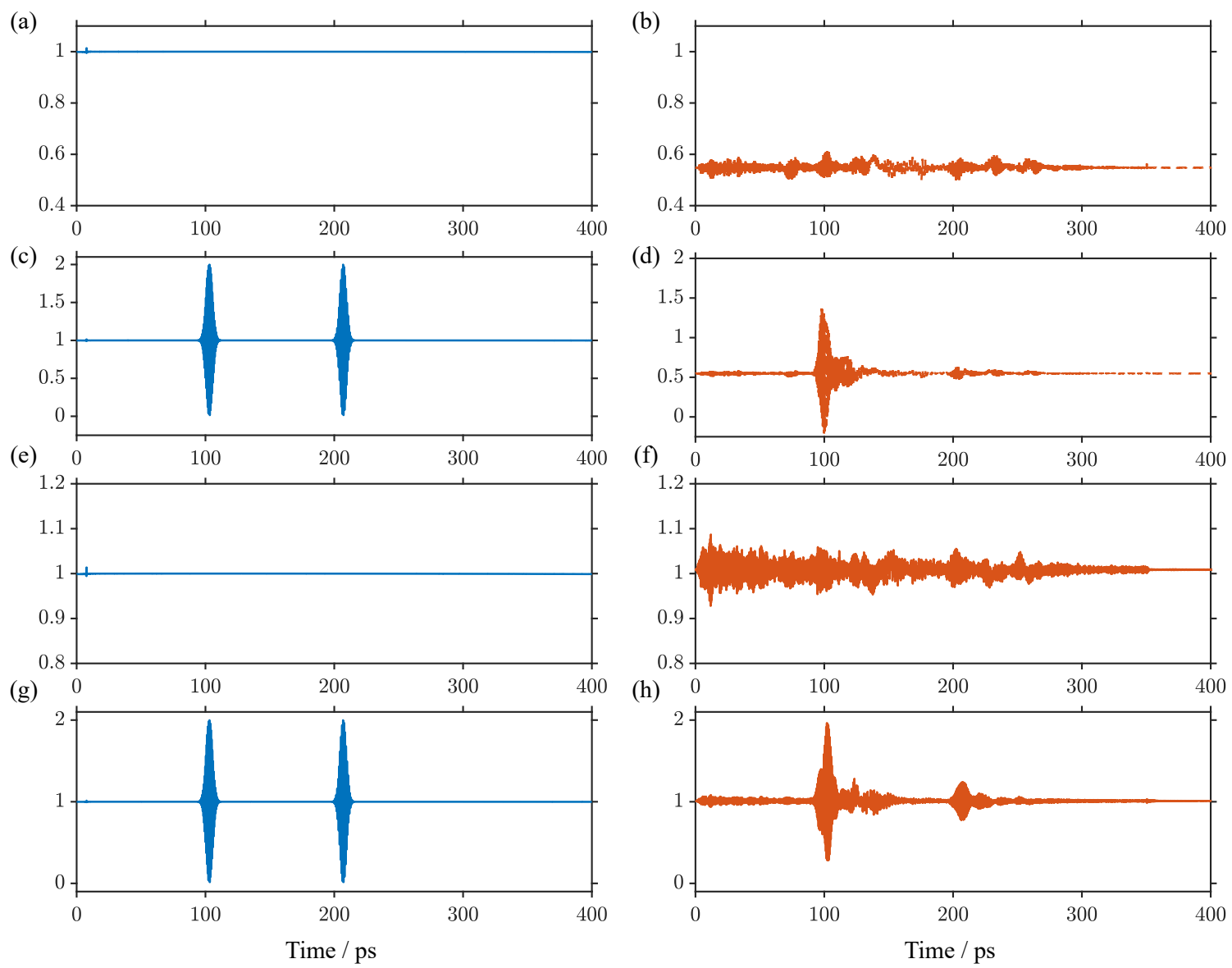

Figure 4. Target signal (left side) signal for (a,e) normal-normal and (c,g) normal-diseased samples and (right side) the signal obtained $(\mathrm{b}, \mathrm{f})$ in the presence of normal-normal and $(\mathrm{d}, \mathrm{h})$ normal-diseased samples. Subplots (a,b,c,d) are obtained using randomly picked tuning parameters $T_{F}=0.12 \mathrm{ps}$ and $T_{T}=6 \mathrm{ps}$ that are non-optimal. They show an $N M S E=21 \%$. On the other hand, subplots $(\mathrm{e}, \mathrm{f}, \mathrm{g}, \mathrm{h})$ are obtained using the optimum parameters $T_{F}=0.1 \mathrm{ps}$ and $T_{T}=7.7 \mathrm{ps}$ and this results in a NMSE of only $0.22 \%$.

\subsection{Validation and the impact of tuning parameters}

To demonstrate the impact of tuning parameters, Fig. 4 shows the validation results of the photonic reservoir system to predict the sample in the test channel. In the validation session, the read-out layer is supplied with a new set of reservoir activation signals by introducing a random white noise to the original FDTD signal. For present demonstration purposes $1 \%$ noise with respect to the reservoir activation signals mean is numerically introduced. Figure 4 shows (left side) the target signal for (a, e) normal-normal and (c, g) normal-diseased samples and (right side) the signal obtained for $(b, f)$ in the presence of normal-normal and $(\mathrm{d}, \mathrm{h})$ normaldiseased samples. Subplots (a, b, c, d) are obtained using randomly picked tuning parameters $T_{F}=0.12 \mathrm{ps}$ and $T_{T}=6$ ps that are non-optimal and yield an NMSE $=21 \%$. On the other hand subplots $(\mathrm{e}, \mathrm{f}, \mathrm{g}, \mathrm{h})$ are obtained using the optimum parameters $T_{F}=0.1 \mathrm{ps}$ and $T_{T}=7.7 \mathrm{ps}$ which result in a NMSE of only $0.22 \%$. We emphasise once again that further optimisation can be performed, for example by using other target signal functions, temporal-separation between Gaussian pulses of the incident signal, or by engineering the size of the D-shaped cavity to provide a high-Q factor cavity (i.e. long-decay time).

\section{CONCLUSIONS}

There is a need to develop an objective, accurate test that would allow screening and diagnosis of disease in a rapid, non-invasive and reliable way. The Photonic Reservoir Computer (PhRC) discussed in this paper can be 
trained to undertake bespoke information processing tasks. Simlulaitons have shown that the PhRC provides an important and timely means by which to achieve, all-optically, the demanding signal processing tasks required by MIR spectro-microscopy using existing and emerging integrated MIR photonics technology platforms; the present simulations showed that a chaotic micro-resonator is a suitable platform for the optical implementation of such an Artificial Neural network. The prospect of discriminating the subtle differences in temporal light signals in real-time brings hopes for a real-time diagnostic tool closer to reality.

\section{REFERENCES}

[1] Baehr-Jones, T., Spott, A., Ilic, R., Spott, A., Penkov, B., Asher, W., and Hochberg, M., "Silicon-onsapphire integrated waveguides for the mid-infrared," Optics Express 18(12), 12127-12135 (2010).

[2] Radosavljevic, S., Kuyken, B., and Roelkens, G., "Efficient $5.2 \mu \mathrm{m}$ wavelength fiber-to-chip grating couplers for the ge-on-si and ge-on-soi mid-infrared waveguide platform," Optics express 25(16), 19034-19042 (2017).

[3] Penadés, J. S., Alonso-Ramos, C., Khokhar, A., Nedeljkovic, M., Boodhoo, L., Ortega-Moñux, A., MolinaFernández, I., Cheben, P., and Mashanovich, G., "Suspended soi waveguide with sub-wavelength grating cladding for mid-infrared," Optics letters 39(19), 5661-5664 (2014).

[4] McKinney, S. M., Sieniek, M., Godbole, V., Godwin, J., Antropova, N., Ashrafian, H., Back, T., Chesus, M., Corrado, G. C., Darzi, A., et al., "International evaluation of an ai system for breast cancer screening," Nature 577(7788), 89-94 (2020).

[5] Goldenberg, S. L., Nir, G., and Salcudean, S. E., "A new era: artificial intelligence and machine learning in prostate cancer," Nature Reviews Urology, 1 (2019).

[6] Petersen, C. R., Prtljaga, N., Farries, M., Ward, J., Napier, B., Lloyd, G. R., Nallala, J., Stone, N., and Bang, O., "Mid-infrared multispectral tissue imaging using a chalcogenide fiber supercontinuum source," Optics letters 43(5), 999-1002 (2018).

[7] Lloyd, G. R. and Stone, N., "Method for identification of spectral targets in discrete frequency infrared spectroscopy for clinical diagnostics," Applied spectroscopy 69(9), 1066-1073 (2015).

[8] Borondics, F., Jossent, M., Sandt, C., Lavoute, L., Gaponov, D., Hideur, A., Dumas, P., and Février, S., "Supercontinuum-based fourier transform infrared spectromicroscopy," Optica 5(4), 378-381 (2018).

[9] Jaeger, H., "Short term memory in echo state networks," GMD-Forschungszentrum Informationstechnik $\mathbf{5}$ (2001). http://www.faculty.jacobs-university.de/hjaeger/pubs/STMEchoStatesTechRep.pdf.

[10] Lukoševičius, M., Jaeger, H., and Schrauwen, B., "Reservoir computing trends," KI-Künstliche Intelligenz 26(4), 365-371 (2012).

[11] Katumba, A., Freiberger, M., Laporte, F., Lugnan, A., Sackesyn, S., Ma, C., Dambre, J., and Bienstman, P., "Neuromorphic computing based on silicon photonics and reservoir computing," IEEE Journal of Selected Topics in Quantum Electronics 24(6), 1-10 (2018).

[12] Vandoorne, K., Dierckx, W., Schrauwen, B., Verstraeten, D., Baets, R., Bienstman, P., and Van Campenhout, J., "Toward optical signal processing using photonic reservoir computing," Optics express 16(15), 11182-11192 (2008).

[13] Vandoorne, K., Dambre, J., Verstraeten, D., Schrauwen, B., and Bienstman, P., "Parallel reservoir computing using optical amplifiers," IEEE transactions on neural networks 22(9), 1469-1481 (2011).

[14] Phang, S., Sewell, P. D., Vukovic, A., and Benson, T. M., "The optical reservoir computer: A new approach to a programmable integrated optics system based on an artificial neural network," in [Integrated Optics: Recent Advances and Prospect], The Institution of Engineering and Technology (IET) (2019).

[15] Freiberger, M., Katumba, A., Bienstman, P., and Dambre, J., "Training passive photonic reservoirs with integrated optical readout," IEEE transactions on neural networks and learning systems (2018).

[16] Mesaritakis, C., Papataxiarhis, V., and Syvridis, D., "Micro ring resonators as building blocks for an alloptical high-speed reservoir-computing bit-pattern-recognition system," JOSA B 30(11), 3048-3055 (2013).

[17] Appeltant, L., Soriano, M. C., Van der Sande, G., Danckaert, J., Massar, S., Dambre, J., Schrauwen, B., Mirasso, C. R., and Fischer, I., "Information processing using a single dynamical node as complex system," Nature communications 2, 468 (2011).

[18] Ellis, D. I. and Goodacre, R., "Metabolic fingerprinting in disease diagnosis: biomedical applications of infrared and raman spectroscopy," Analyst 131(8), 875-885 (2006).

[19] Lumerical, "FDTD solutions," (2016). https://www.lumerical.com/products/. 